\title{
STUDY OF THE DISAPPEARANCE OF CONGO RED FROM THE BLOOD OF NON-AMYLOID SUBJECTS AND PATIENTS WITH AMYLOIDOSIS
}

\author{
BY PAUL N. UNGER, MORRIS ZUCKERBROD, GUSTAV J. BECK AND \\ J. MURRAY STEELE WITH THE TECHNICAL ASSISTANCE OF \\ YETTA POROSOWSKA \\ (From the Third [New York University] Medical Division, Goldwater Memorial Hospital, \\ Welfare Island, New York)
}

(Received for publication July 29, 1947)

In a case suspected clinically of having amyloidosis because of the presence of a chronic infection, large liver and spleen, and moderate albuminuria, a Congo Red test done by the current standard method (1) proved to be negative according to present interpretation (60 per cent of the dye was removed from the blood at the end of 60 minutes). A moderate amount of amyloid was, however, shown to be present by biopsy of the liver. For this and other considerations it was decided to review the various methods of carrying out the test and the criteria employed in interpretation.

In 1923 Bennhold (2) described a method for the laboratory diagnosis of amyloidosis by the intravenous injection of $10 \mathrm{cc}$. of a 1 per cent solution of Congo Red. ${ }^{1} \mathrm{He}$ regarded the disappearance of more than 60 per cent of the Congo Red at the end of an hour as presumptive evidence of amyloid disease. Subsequent investigators (Friedman and Auerbach [1], Taran and Eckstein [3], Lipstein [4], Auerbach and Stemmerman [5]), modified Bennhold's original method because they found that many falsely positive results were obtained using his criteria. They suggested that at least 90 per cent of the dye be absorbed in an hour before making the diagnosis of amyloidosis.

In 1942, Taran and Eckstein (3) found that the 4-minute specimen was frequently too light in color to be relied upon as the standard or 100 per cent specimen. They recommended that a 2minute specimen be taken in addition to the 4minute and 1-hour samples, advising that the 2minute specimen be used as the standard for comparison (100 per cent). They gave $1 \mathrm{cc}$. of a 1 per cent Congo Red solution for each 10 pounds of body weight. In order to avoid errors due to

\footnotetext{
1 The Congo Red used in this study was furnished by the C. F. Kirk Co., New York City.
}

hemolysis, they precipitated the liberated hemoglobin with acetone. Some time earlier, Friedman and Auerbach (1) had used 95 per cent alcohol as the hemoglobin-precipitating agent.

Harmon and Kernwein (6) in 1942 modified the Congo Red test by using known standard solutions of Congo Red for comparison. They used isotonic sodium oxalate solution as the anti-coagulant, performing the determinations on plasma. They tried to avoid hemolysis by careful collection of specimens rather than by correcting for it by precipitation of the hemoglobin. It should be noted, however, that they also used a 4-minute specimen as their 100 per cent standard.

There are at least 3 significant sources of error in all the methods previously described. The first lies in the assumption. that the injected dye is completely mixed at the end of 2 or 4 minutes. That this is not true for most persons has been amply demonstrated by Gregersen (7), Gibson and Evans (8), Price and Longmire (9). Using Evans blue, these investigators showed that at least $71 / 2$ to 20 minutes are required for complete mixing in normal people and even longer in persons with slower circulations. The second important source of error lies in the assumption that little or no absorption of the injected Congo Red takes place in amyloidosis before the 2- or 4-minute specimen is obtained. Evidence presented in this paper suggests that this assumption may well be the main source of error in the so-called "falsenegative" tests (Figure 1). The third source of error is the personal equation which enters into the color matchings whenever optical colorimeters are used.

In an effort to estimate the magnitude of these errors and correct for them, it was decided to study the behavior of intravenously administered Congo Red by determining dye concentration in the 


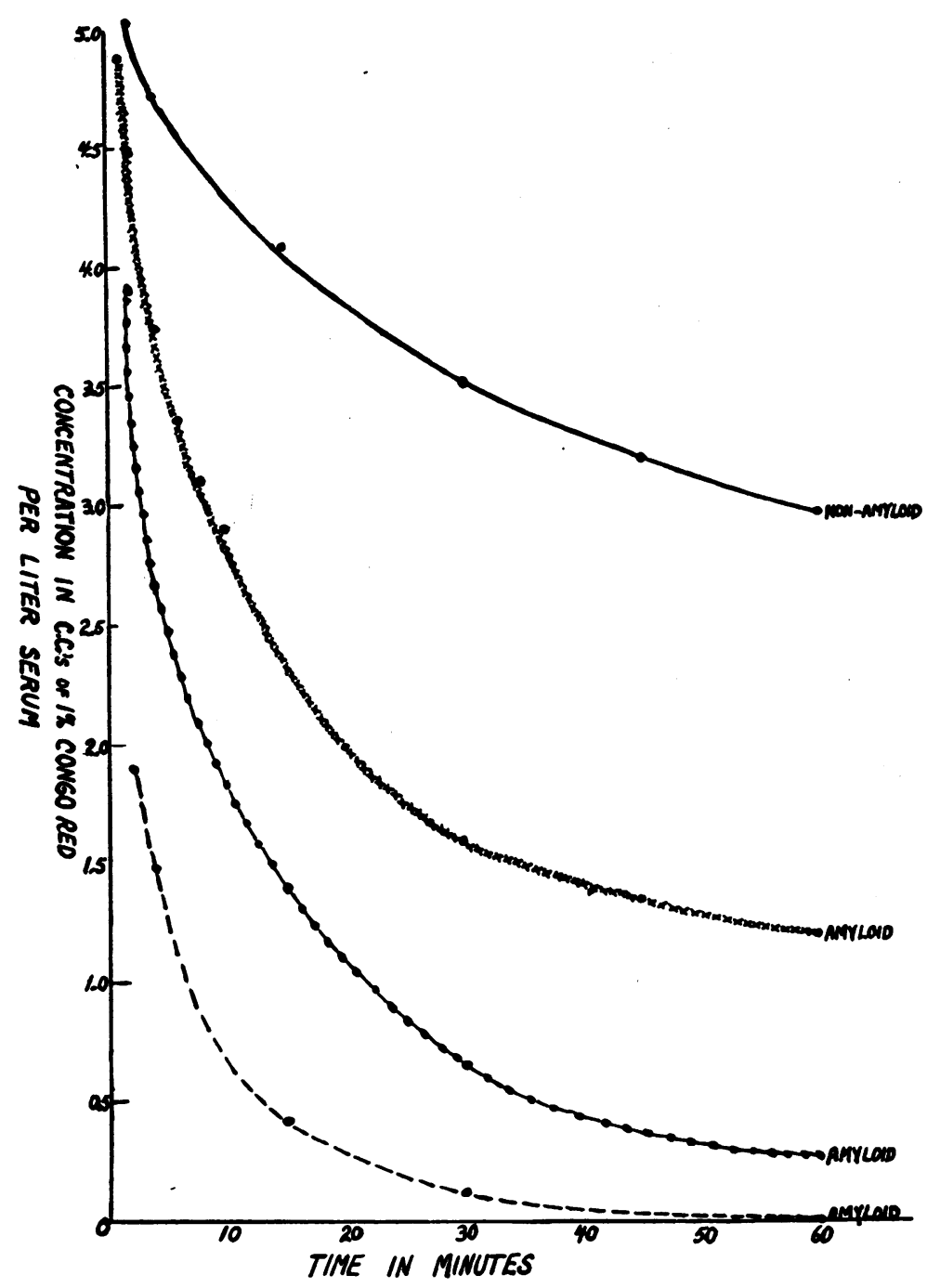

Fig. 1. Disappearance Curves in Non-Amyloid and Amylord Subjects

serum at frequent intervals during the course of an hour after its administration. The urine was also collected during this hour and Congo Red, if present, was quantitatively assayed. All determinations were done on the Coleman spectrophotometer. The rate of disappearance of Congo Red from the blood stream in normal persons as well as in persons with amyloid disease was studied.

Because neither a 2- nor 4-minute specimen appeared to be reliable for use as the 100 per cent standard, initial effort was directed toward finding a method for determining the theoretical concentration of Congo Red in the blood following intravenous injection of a known amount. Several means were open to study.

One was obviously the determination of plasma volumes, which would at once permit calculation of the theoretical concentration of Congo Red at zero time with complete mixing. Using $\mathrm{T}-1824$ (Evans blue) in non-amyloid subjects, satisfactory measurements of blood volume were obtained. In patients with amyloid disease, however, abnormally high plasma volumes were found. For example, in patients who weighed 90 to 110 pounds, plasma volumes of 4100 to $5200 \mathrm{cc}$. were obtained. One explanation was that since Evans blue is a vital dye, it was likely that some of it had been 
fixed by amyloid tissue. On staining amyloid tissue obtained at post-mortem examination with Evans blue, it was found that such was the case. Much more of the dye is fixed by this tissue than by normal tissue.
A rough method for determining plasma volume is the one based on surface area or body weight. From previous reports $(8,10,11,12)$ this is about $44 \mathrm{cc}$. per kilogram body weight, or 1628 cc. per square meter surface area. However, these

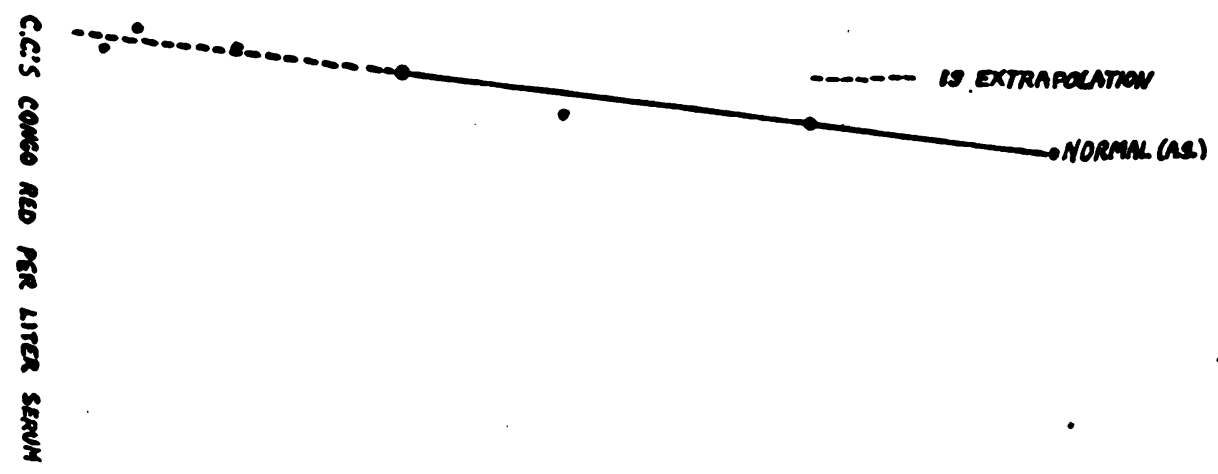

(1)

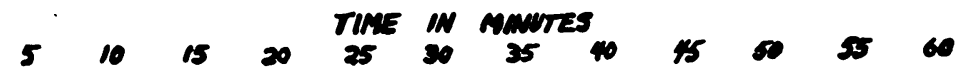

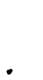
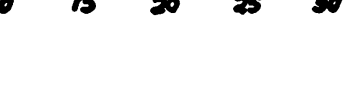

35 
figures represent average values for normal individuals between the ages of 17 and 89 and variations that occur in disease may introduce significant errors. A third method for measuring plasma volume is by introduction of radioactive material in the form of red blood cells, but this means was not at the moment available.

An alternate method for determining the theoretical concentration of Congo Red is the extrapolation to zero time of a curve which is plotted from a large number of determinations. In normal persons it was found, in an effort to determine the theoretical concentration, that a straight line is obtained when points between 10 and 60 minutes are plotted. Points between 2 and 10 minutes are variable and consequently unreliable because mixing is incomplete.

In amyloid disease, however, the curve on semilogarithmic paper was not straight. The theoretical concentration could best be approximated by extrapolation derived from that portion of the curve obtained from values in the first 10 minutes. The curve in this portion was more nearly straight probably because rapid disappearance of the dye tended to mask the vagaries of mixing (Figure 2 ).

\section{METHOD}

1. Preparation of standard Congo Red absorption curve. $1 \mathrm{cc}$. of a 1 per cent Congo Red solution is diluted to 500 cc. with a $1: 1$ acetone water solution. This is used as a stock solution. Solutions of $10,25,371 / 2,50$ and 75 per cent are prepared and their transmission value, determined in a Coleman Junior Spectrophotometer, is plotted against concentration on semilogarithmic paper. This yields a straight-line relationship up to 75 per cent concentration. Beyond this point there is a slight change in the slope of the line. By diluting the unknown specimens, one can keep within the 10 to 75 per cent range without difficulty. It was found that identical readings with the acetone water diluent were given by using distilled water alone as the diluting agent in the preparation of the standard. A wave length of 500 was found to be the most satisfactory, and this is the one generally recommended. It is important to prepare the standard and perform the tests with the same lot of Congo Red, because of the known variations in different batches of organic dyes.

2. Technique of the test. The Congo Red determination is done after a fast of at least 8 to 12 hours to avoid the lipemia which interferes with colorimetric readings. A blood specimen to serve as the blank control is taken prior to the injection of $1 \mathrm{cc}$. of 1 per cent Congo Red solution per 10 pounds of body weight. $10 \mathrm{cc}$. blood samples are removed from the opposite arm at $2,4,6,8$,
$10,20,30,45$ and 60 minutes. The needle may remain in place for the withdrawal of the first 5 specimens without using anti-coagulants.

The blood is permitted to stand at room temperature for 1 hour and then placed in the refrigerator for a half hour to facilitate separation of the clot. The serum is separated by centrifugation and 2-cc. amounts are placed in centrifuge tubes. Depending upon the intensity of color, gauged by the naked eye, $1: 4$ dilutions with acetone at room temperature are prepared for the stronger solutions, and $1: 2$ dilutions for the weaker ones. The blank is treated in an identical manner. All of the serum specimens from a given patient are treated in the same way.

After the addition of the acetone, the tubes are stoppered, vigorously shaken to insure complete mixing and thorough precipitation of the hemoglobin and protein. The specimens are then centrifuged for 10 minutes at 10,000 r.p.m. to sediment the precipitate. A crystal-clear solution of Congo Red is regularly the result.

Serum rather than plasma was used for 3 reasons. First, because the use of $50 \mathrm{mgm}$. of dry sodium oxalate per $100 \mathrm{cc}$. of whole blood significantly changes the hematocrit reading by shrinking the RBCs (13). Second, isosmotic sodium oxalate would introduce a source of error by dilution in the concentration of the dye. Third, the use of heparin introduces a yellow color into the plasma.

That the process of clotting does not remove a significant amount of dye from the serum is shown by comparing the dye concentration in serum and plasma prepared from the same whole blood specimens.

$\begin{gathered}\text { Concentration } \\ \text { in serum }\end{gathered}$
3.88
3.72
3.52
3.36
3.20

$\begin{gathered}\text { Concentration } \\ \text { in plasma }\end{gathered}$
3.32
3.36
3.36
3.20
2.92

Dry sodium oxalate was used as the anti-coagulant, and the resulting shrinkage of the RBCs and consequent increase in plasma volume account for the lower dye concentration in the plasma compared with the serum. Keith, Rowntree and Geraghty (14) showed that the RBCs do not take up any of the vital dyes.

Nor does the precipitation of serum proteins and hemoglobin resulting from hemolysis remove any dye. This was demonstrated by quantitative recovery of known amounts of added dye from serum and plasma solutions after protein precipitation.

The supernatant fluid is decanted into colorimetric tubes and the colorimeter set at 100 with the blank in position. Readings are then taken at a wave length of 500 . It is quite safe to allow the serum to stand in stoppered tubes in a refrigerator for several days without any alteration of the color intensity as determined by the spectrophotometer.

3. Calculation of results. Results are expressed in the form of cc. of 1 per cent Congo Red per liter of serum. The transmission values of the various specimens are converted into concentration by the prepared curve of 
standard dilutions. These values are then multiplied by the dilution used in preparing the protein-free solution.

Example: The colorimeter reading in per cent transmission is 43 . The concentration obtained from the standard dilution curve is $1.34 \mathrm{cc}$. per liter. Since a $1: 4$ dilution was made in preparing the protein filtrate, the concentration of Congo Red in the serum is, therefore, $5.36 \mathrm{cc}$. of 1 per cent Congo Red per liter.

\section{Excretion of dye in urine}

Some investigators have stated (6), and it is a current concept that under certain conditions, intravenous injection of Congo Red may be followed by the appearance of the dye in the urine. When this occurs, the test is supposed to lose value. To investigate the validity of these statements, a method for the extraction and quantitative estimation of Congo Red in the urine was worked out. $^{2}$

An extracting reagent is prepared by making a 1 per cent solution of quinine sulphate in chloroform or ethylene dichloride. This solution must be freshly prepared because rapid discoloration occurs. This is filtered and 10 cc. of $1 \mathrm{~N} \mathrm{NaOH}$ is added to each $100 \mathrm{cc}$. of solution. To $30 \mathrm{cc}$. of urine, $5 \mathrm{cc}$. of saturated $\mathrm{NaHCO}_{3}$ is added, to which, in turn, is added $35 \mathrm{cc}$. of the extracting reagent. The mixture is vigorously shaken and then centrifuged. The process is repeated until all of the dye has gone into the extracting solution. The supernatant layer is then aspirated. To $10 \mathrm{cc}$. of the quinine Congo Red solution 3 cc. of $1 \mathrm{~N} \mathrm{NaOH}$ is then added to bring the $\mathrm{pH}$ of the solution to 8 or 8.5 , at which $\mathrm{pH}$ the dye goes into the aqueous phase when the mixture is vigorously shaken and centrifuged. The colored solution is then read in the spectrophotometer at a wave length of 500 against a urine blank and a distilled water blank.

It has been found that this method is accurate enough to detect $1 / 10,000 \mathrm{cc}$. of Congo Red per $100 \mathrm{cc}$. of urine. For ready comparison, a series of standards with known concentrations of the dye can be set up. In the series of Congo Red determinations reported in this paper, no urine was found which contained more than a faint trace of dye, despite the presence of large amounts of albumin, too little to affect the concentration in the blood.

\section{Characteristics of the disappearance curve}

It is apparent that in the normal subject 2 mechanisms are operating; namely, mixing, which occurs in the first 8 to 15 minutes, and removal of the dye from the blood stream presumably by the reticulo-endothelial system (Figure 2). In the presence of amyloid, the mixing factor is obscured by the very rapid removal of the dye during the first 30 minutes after injection (Figure 2). Removal during the second 30 minutes is much slower. It is presumed that this initial phase of rapid removal is referable to fixation of the injected dye in the amyloid

${ }^{2} \mathrm{We}$ are indebted to Dr. B. B. Brodie, N. Y. U. Research Service, Goldwater Memorial Hospital, for developing these procedures. tissue. In the second 30 minutes, the slower rate of removal approaches the disappearance rate occurring in normal individuals.

The greatest differences between the curves of normal individuals and those with deposits of amyloid occur in the first 30 minutes. It would be reasonable, therefore, to base a Congo Red test on this time interval. Results can be estimated in 2 ways. One, serum concentration of dye is plotted against time on semi-logarithmic paper, and the resulting line is extrapolated back to zero time (Figure 2). The concentration obtained at zero time is used as the standard with which to compare the value at 30 minutes, and the percentage of dye which has been absorbed or lost from the blood stream is calculated. A simpler but somewhat less accurate method is to calculate the concentration of dye at zero time on the basis of a serum volume deduced from body weight or surface area relationships.

The plasma or serum volume in normal individuals ranges from 1624 to $1680 \mathrm{cc}$. per square meter of surface area with an average value of 1628 , and 40 to $49 \mathrm{cc}$. per kilogram, with an average of $44(8,10,11,12)$. In sthenic individuals the larger values are used. The $C_{0}$ (concentration at zero time) is compared with a 30minute specimen as previously noted. The advantage of this method is that it avoids numerous venipunctures and processing of numerous blood specimens. Evidently it cannot be applied to patients with cardiac failure, dehydration or other situations where a significant deviation of the plasma volume from the normal is to be expected.

In this study, the $C_{0}$ was obtained by the extrapolation of curves of concentration. However, it was found that the quantities of Congo Red absorbed after 30 minutes, calculated from extrapolation of curves to zero time or estimated on body weight or surface area, were so nearly equal in value that for practical purposes the simpler method might be used. The validity of this statement needs to be verified by a larger number of tests in both normal and amyloid subjects than is now presented by the table below comparing values obtained from extrapolation and estimation in normal and amyloid subjects.

\begin{tabular}{l|c|c|c|c|c}
\hline \hline Patient & $\begin{array}{c}\text { Surface } \\
\text { area in } \\
\text { sq. meters }\end{array}$ & $\begin{array}{c}C_{0} \\
\text { calcu- } \\
\text { lated }\end{array}$ & $\begin{array}{c}C_{0} \\
\text { extrapo- } \\
\text { lated }\end{array}$ & \multicolumn{2}{|c}{$\begin{array}{c}\text { Per cent removed } \\
\text { in 30 min. }\end{array}$} \\
\cline { 4 - 6 } & & Calc. $C_{0}$ & Extrap. $C_{0}$ \\
\hline B. R. & 1.88 & 5.70 & 5.75 & 14 & 15 \\
K. Z. & 1.94 & 5.50 & 5.40 & 13 & 12 \\
M. Z. & 1.87 & 5.25 & 5.15 & 15 & 13 \\
M. M. & 1.58 & 5.20 & 5.70 & 68 & 71 \\
M. W. & 1.42 & 5.90 & 5.20 & 73 & 69 \\
J. M. & 1.67 & 4.90 & 4.00 & 87 & 84 \\
\hline
\end{tabular}

\section{RESULTS}

Using the method outlined, the disappearance of Congo Red from the blood was studied in 74 subjects, 10 of whom were healthy young adults, 52 with rheumatoid arthritis, and the remaining 
12 with various diseases. The results are given in terms of percentage of dye removed from the blood in 30 minutes (Figure 3 ). Sixty-eight cases showed from 4 per cent to 32 per cent removal of injected dye in 30 minutes. None of these patients had any clinical evidence of amyloidosis. In the remaining 6 patients, all with rheumatoid arthritis, 68 per cent to 96 per cent of the injected dye was removed from the blood in 30 minutes. All of these patients showed confirmatory clinical findings of amyloidosis. The diagnosis was verified by liver biopsy in 2 patients where consent to the procedure was given. One had a Congo Red absorption of 69 per cent and the other 96 per cent.

In the subjects with amyloidosis, Congo Red was not found in the urine. In only 5 of the other subjects did Congo Red appear in the urine, and then in such small amounts as to be insignificant from a quantitative point of view.

A frequency distribution curve of the amounts of dye removed from the blood in 30 minutes shows that the patients with evidence of amyloidosis fall in an entirely different segment from non-amyloid subjects (Figure 4). Of interest is the fact that
1 of the patients with amyloid disease showed, on liver biopsy, deposition of amyloid in only several small blood vessels. He absorbed only 69 per cent of the dye at the end of 30 minutes.

The range of values in non-amyloids at $30 \mathrm{~min}$ utes varies from 4 per cent to 32 per cent removal, with an average of 17 per cent (Figure 3 ). This compares with a range of 15 per cent to 51 per cent and an average of 31 per cent for this same group at 60 minutes.

In the amyloids, the range of values at $30 \mathrm{~min}$ utes is from 68 to 96 , with an average of 80 per cent. At 60 minutes the range is 77 to 100 with an average of 88 per cent. The difference of 8 per cent between the average values at 60 and $30 \mathrm{~min}$ utes in amyloids supports the concept that the removal of dye by amyloid tissue occurs largely in the first 30 minutes.

The data presented here indicate that the 2 - or 4-minute blood specimen may be too dark (because of incomplete mixing) or too light in color (because of rapid fixation by amyloid) to be reliable as a 100 per cent standard for comparison. A better value is obtained from extrapolation of

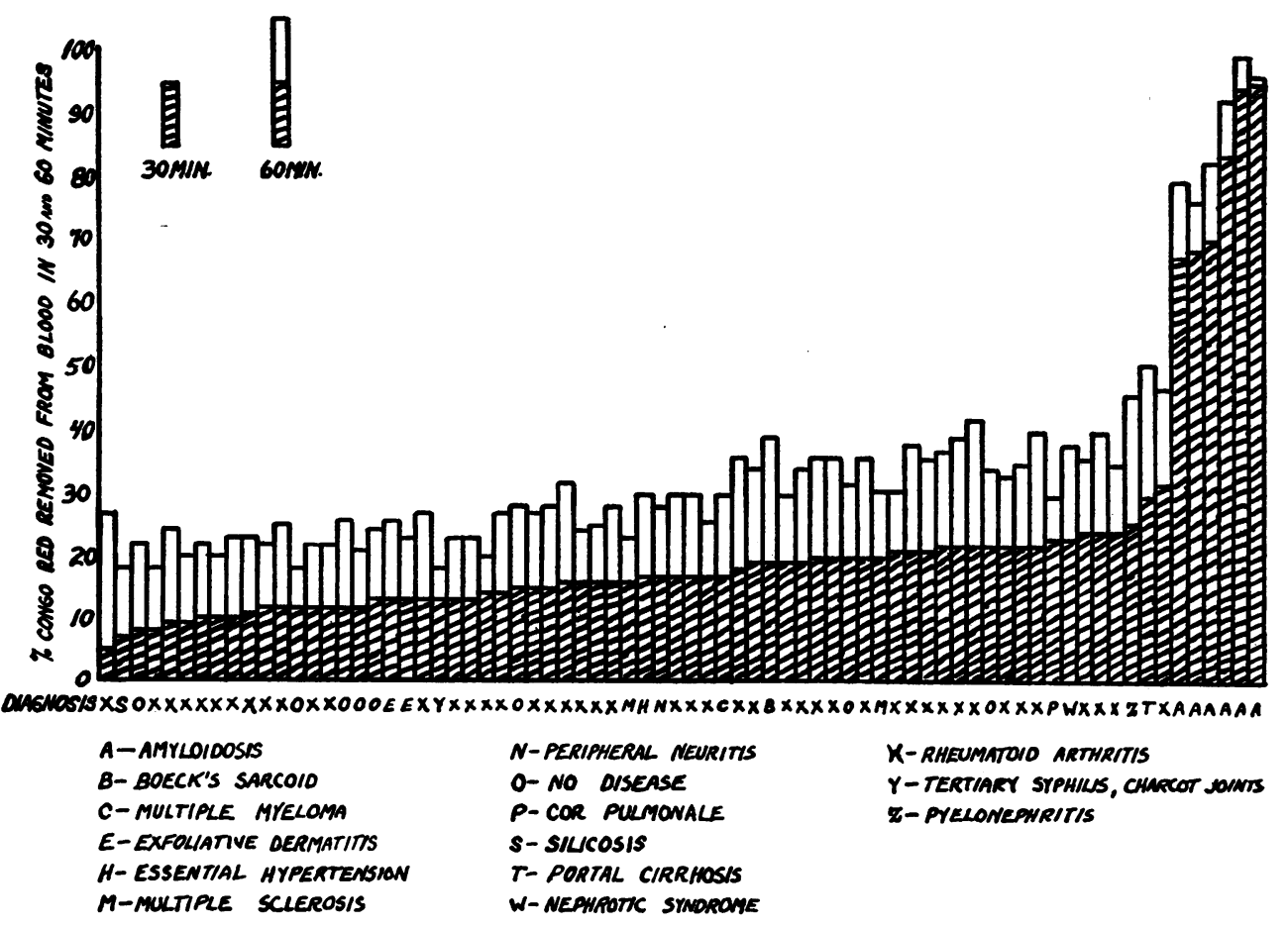

Fig. 3. Disappearance of Congo Red from Blood Studied in 74 Subjects Results given in terms of percentage of dye removed in 30 minutes 


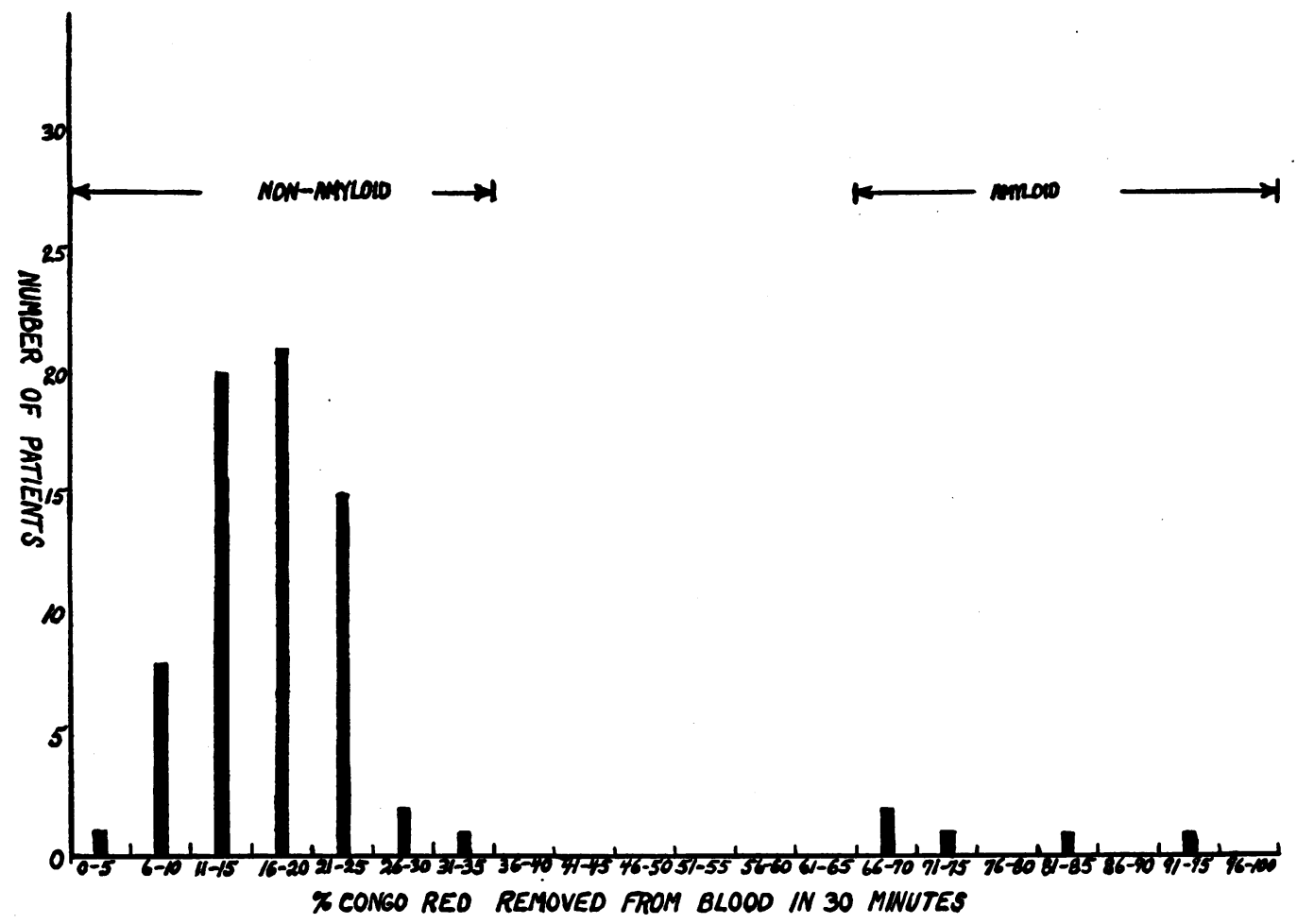

Fig. 4. Congo Red Removed from Blood in 30 Minutes in Non-Amyloids and Amyloids

the curve of disappearance or calculation of blood volume from surface area or body weight. Because the greatest difference in the curves of removal of Congo Red from the blood of nonamyloid and amyloid patients exists at about 30 minutes after injection, this time interval seems the one of choice as the end point of the test. Since the normal range extended as high as 32 per cent removal of the dye from the blood at the end of 30 minutes, more than 35 per cent removal in this time interval is presumptive evidence of amyloidosis. However, a larger series of normal persons is needed before the critical level is decided upon.

\section{CONCLUSION}

1. The use of Congo Red for testing for the presence of amyloidosis has been simplified and the accuracy increased by

(a) calculating the theoretical initial concentration rather than using a 2- or 4-minute specimen for comparison,

(b) using $\mathbf{3 0}$ minutes rather than an hour as the end point.
2. T-1824 (Evans Blue) cannot be used for the calculation of blood volume in amyloidosis, because amyloid tissue fixes this dye also.

\section{BIBLIOGRAPHY}

1. Friedman, M. M., and Auerbach, O., An improved Congo Red test for amyloidosis. J. Lab. \& Clin. Med., 1935, 21, 93.

2. Bennhold, H., Excretion of intravenously injected Congo Red in different diseases, especially amyloidosis. Deutsches Arch. f. klin. M., 1923, 142, 32.

3. Taran, A., and Eckstein, A., The standardization of the Congo Red test for amyloidosis. Am. J. M. Sc., 1942, 203, 246.

4. Lipstein, S., An evaluation of the Congo Red test for amyloidosis. Am. J. M. Sc., 1938, 195, 205.

5. Stemmerman, M., and Auerbach, $O$., The value and limitations of the Congo Red test for amyloidosis. Am. J. M. Sc., 1944, 208, 305.

6. Harmon, P. H., and Kernwein, G., Congo Red test for amyloid disease; quantitative technic. Arch. Int. Med., 1942, 70, 416.

7. Macleod's Physiology in Modern Medicine; Distribution and regulation of water in the body, Gregersen, M. I. C. V. Mosby Co., St. Louis, 1938, Ed. 8.

8. Gibson, J. G., 2nd, and Evans, W. A., Jr., Clinical studies of the blood volume. J. Clin. Invest., 1937, 16, 317. 
9. Price, P. B., and Longmire, W. P., The use of T-1824 in plasma volume determinations. Bull. Johns Hopkins Hosp., 1942, 71, 51.

10. Brines, J. K., Gibson, J. G., 2nd, and Kunkel, P., The blood volume in normal infants and children. J. Pediat., 1941, 18, 447.

11. Stewart, J. D., and Rourke, G. M., On the measurement of extracellular fluid volume with thiocyanate and body fluid analyses in 33 normal individuals. J. Lab. \& Clin. Med., 1941, 26, 1383.
12. Davis, L. J., The determination of blood volume in man with Evans blue (T-1824). Edinburgh M. J., 1942, 49, 465.

13. Hooper, J., Jr., Smith, H. P., Belt, A. E., and Whipple, G., Blood volume studies: experimental control of a dye blood volume method. Amer. J. Physiol., 1920, 51, 205.

14. Keith, N. M., Rowntree, L. G., and Geraghty, J. T., A method for the determination of plasma and blood volume. Arch. Int. Med., 1915, 16, 547. 